\title{
REACTION-DIFFUSION FRONT SPEEDS IN SPATIALLY-TEMPORALLY PERIODIC SHEAR FLOWS*
}

\author{
JIM NOLEN ${ }^{\dagger}$ AND JACK XIN ${ }^{\ddagger}$
}

\begin{abstract}
We study the asymptotics of two space dimensional reaction-diffusion front speeds through mean zero space-time periodic shears using both analytical and numerical methods. The analysis hinges on traveling fronts and their estimates based on qualitative properties such as monotonicity and a priori integral inequalities. The computation uses an explicit second order upwind finite difference method to provide more quantitative information. At small shear amplitudes, front speeds are enhanced by an amount proportional to shear amplitude squared. The proportionality constant has a closed form expression. It decreases with increasing shear temporal frequency and is independent of the form of the known reaction nonlinearities. At large shear amplitudes and for all reaction nonlinearities, the enhanced speeds grow proportional to shear amplitude and are again decreasing with increasing shear temporal frequencies. The results extend previous ones in the literature on front speeds through spatially periodic shears and show front speed slowdown due to shear direction switching in time.
\end{abstract}

Key words. fronts, space-time periodic shears, speed enhancement, asymptotics, computation

AMS subject classifications. 35K57, 41A60, 65D30

DOI. $10.1137 / \mathrm{S} 1540345902420234$

1. Introduction. Front propagation in heterogeneous media appears in many scientific disciplines such as combustion of premixed flames, reactive pollutant transport in porous media, and invasion of biological species, just to name a few [7], [33], [24], [30]. Though heterogeneities are dominantly spatial in the latter two areas of application, in combustion, especially turbulent premixed flame problems, a wide range of spatial and temporal scales can arise in the multiscale environment (i.e., fluid advection) where flames are moving; see, e.g., [13], [19], [20], and the references therein. The large time propagation speed of a flame front is an upscaled quantity depending on both flow and chemistry, and is often much larger than a pure reaction-diffusion front speed (called laminar speed). It is open in general how to mathematically establish the existence and properties of the upscaled speed, denoted by $c_{*}$, from now on, when the flow velocity has turbulence spectrum. For related data and empirical laws, see [22], [32], [16], among others.

When the flow structures and scales are simplified, much recent progress has been made in analyzing the asymptotic behaviors of upscaled front speeds in the regimes of weak and strong advection. To introduce some of the existing results related to our work here, let us consider the scalar reaction-diffusion-advection equation (R-D) of the form

$$
u_{t}=\Delta_{y} u+\delta W \cdot \nabla u+f(u), \quad y=\left(y_{1}, y_{2}\right) \in R^{2},
$$

where $W=\left(0, w\left(y_{1}, t\right)\right)$, a mean zero space-time periodic shear flow, $\delta>0$ being a

*Received by the editors December 24, 2002; accepted for publication (in revised form) June 9, 2003; published electronically October 10, 2003. This research was partially supported by NSF grant ITR-0219004.

http://www.siam.org/journals/mms/1-4/42023.html

${ }^{\dagger}$ Department of Mathematics, University of Texas at Austin, Austin, TX 78712 (jnolen@math. utexas.edu).

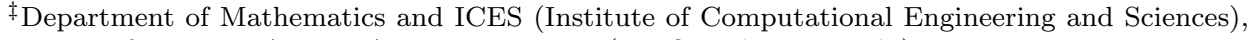
University of Texas at Austin, Austin, TX 78712 (jxin@math.utexas.edu). 
parameter measuring the shear strength; $\Delta_{y}=\partial_{y_{1} y_{1}}^{2}+\partial_{y_{2} y_{2}}^{2}, f(u)$ being the reaction nonlinearity. The nonlinear function $f(u)$ can be

(1) the bistable nonlinearity: $f(u)=u(1-u)(u-\theta), \theta \in(0,1 / 2)$;

(2) the quadratic nonlinearity: $f(u)=u(1-u)$, known as Kolmogorov-PetrovskyPiskunov (KPP);

(3) the higher order KPP nonlinearity: $f(u)=u^{m}(1-u), m \geq 2$, integer $(m=2$, called Zeldovich nonlinearity);

(4) the combustion nonlinearity with ignition temperature cutoff: $f(u)=0, u \in$ $[0, \theta], \theta \in(0,1) ; f(u)>0, u \in(\theta, 1), f(1)=0$;

(5) Arrhenius-type combustion nonlinearity: $f(u)=e^{-E / u}(1-u), E>0$, the activation energy (often large).

When $\delta$ is small, and $w=w\left(y_{1}\right)$ for types (1), (2), and (4) nonlinearities, the large scale front speed obeys a quadratic law of enhancement $\left(\tilde{w}_{y_{1}}=w\left(y_{1}\right), \tilde{w}\right.$ mean zero):

$$
c_{*}=c_{0}\left(1+\frac{1}{2}\left\|\tilde{w}\left(y_{1}\right)\right\|_{2}^{2} \delta^{2}+\text { h.o.t. }\right) \text {, }
$$

$c_{0}$ being the laminar speed; see [21] for perturbation calculations on types (1) and (2), and [15] for variational methods on types (1) and (4). The quadratic law goes back to Clavin and Williams [7] for turbulent flame speed and is challenged in [16] regarding its validity for random flows. A systematic case study of its validity and failure for mean zero stationary ergodic Gaussian random shears was recently presented in [31].

When $\delta$ is large, and $w=w\left(y_{1}, t\right)$ for type (2) nonlinearity (see [9]) or $w=w\left(y_{1}\right)$ for type (4) nonlinearity (see [17]),

$$
c_{*}=O(\delta),
$$

and $c_{*} \sim b_{0} \delta, b_{0}$ being constant, for type (2) and $w=w\left(y_{1}\right)$ [2]. In fact, (1.3) holds for a class of more general time independent flows, percolating flows (which contain at least two infinitely long channels of flow trajectories) [9], [17]. Sublinear enhancement for flows with closed streamlines are studied in [1], [9], [17]. Speed enhancement through large time independent shear and cellular flows is numerically investigated in [25] for types (2) and (4), in agreement with analytical predictions. The linear law $c_{*}=b_{0} \delta+b_{1}$ holds for the large shear case. A simple proof of $O(\delta)$ growth for time independent shears for types (2)-(5), based on equations of traveling fronts, was recently given in [14]. For types (2), (3), and (5), the speed is understood as minimal speed.

In this paper, we analyze the asymptotics of $c_{*}$ for space-time periodic shears based on traveling front solutions of the form $u=\varphi\left(y_{2}-c_{*} t, y_{1}, t\right)$, periodic in $y_{1}$ and $t$. For small $\delta$, combined with a variational calculation of type (2), we derive the quadratic enhancement law, however with a new prefactor that is monotone decreasing in the shear temporal frequency, for $f$ of types (1), (2), and (4). For large $\delta$, we prove the linear growth law (1.3) for types (2)-(5) by extending the method of [14]. A finite difference simulation of (1.1) is performed on a rectangular domain with zero Neumann boundary data and $f$ of types (1)-(4). Numerical results support the quadratic law for small $\delta$ and linear growth law for large $\delta$ for $f$ of types (1)-(5). Moreover, the front speeds at all $\delta$ 's simulated are monotone decreasing in shear temporal frequency and tend to converge for large enough frequencies.

The reduction of front speed enhancement due to temporal oscillation of shear flows was studied earlier for KPP reaction in [18]. The authors parametrized the 
amount of reduction in terms of a nondimensional flame residence time, which is the ratio of shear time period over the front wrinkling time. The latter time scale refers to how long it takes a spatial shear field to distort a flat flame front to reach the enhanced steady speed.

The rest of the paper is organized as follows. Section 2 analyzes quadratic speed enhancement for small shears, and section 3 analyzes linear growth in large shears. Section 4 contains the numerical method and numerical results in agreement with analysis in previous sections. We observe that type (1) fronts behave similarly to type (4) in large shears. Conclusions and remarks on future work are in section 5 . In the appendix, we outline a proof of type (4) traveling front existence and qualitative properties, useful for estimating front speeds.

\section{Front speeds in small amplitude shears.}

2.1. Bistable fronts. Let us first consider bistable fronts in a two-dimensional shear flow. The equation is

$$
u_{t}=\Delta u+W \cdot \nabla u+f(u),
$$

where $W=\left(0, w\left(y_{1}, t\right)\right), \Delta=\partial_{y_{1} y_{1}}^{2}+\partial_{y_{2} y_{2}}^{2}, f(u)=u(1-u)(u-\theta), \theta \in\left(0, \frac{1}{2}\right)$; moreover, $w\left(y_{1}, t\right)$ is a smooth periodic function with period $2 \pi$ in $y_{1}$ and $2 \pi / \omega \equiv T$ in $t$, and its space-time mean equals zero. A traveling wave moving in the $y_{2}$-direction has the form

$$
u=\varphi\left(y_{2}-c_{*} t, y_{1}, t\right) \equiv \varphi\left(s, y_{1}, \tau\right), \quad s=y_{2}-c_{*} t, \quad \tau=t,
$$

$c_{*}$ being the constant large time front speed. This form includes as a special case traveling fronts in time independent shears [3]. Substituting the above into (2.1), we see that $\varphi$ satisfies

$$
\begin{gathered}
\varphi_{s s}+\varphi_{y_{1} y_{1}}+\left(c_{*}+w\left(y_{1}, \tau\right)\right) \varphi_{s}-\varphi_{\tau}+f(\varphi)=0 \\
\varphi\left(-\infty, y_{1}, \tau\right)=0, \quad \varphi\left(+\infty, y_{1}, \tau\right)=1, \quad \varphi(s, \cdot, \cdot)(2 \pi, T) \text {-periodic }
\end{gathered}
$$

and we add a normalization condition

$$
\frac{1}{2 \pi T} \int_{0}^{2 \pi} \int_{0}^{T} \varphi\left(0, y_{1}, \tau\right) d y_{1} d \tau=1 / 2
$$

to fix the translation constant in $s$. Equation (2.2) has unique solution up to a constant translation in $s$; see the appendix.

We are interested here in the effects of $w\left(y_{1}, \tau\right)$ on the speed $c_{*}$. Let us expand $w\left(y_{1}, \tau\right)$ in terms of a small parameter $\delta$,

$$
w\left(y_{1}, \tau\right)=\delta w_{1}\left(y_{1}, \tau\right)+\delta^{2} w_{2}\left(y_{1}, \tau\right)+\cdots
$$

where $w_{i}\left(y_{1}, \tau\right)(i=1,2, \ldots)$ are all mean zero over $[0,2 \pi] \times[0, T]$. Now expand the traveling wave and the speed

$$
\begin{aligned}
& \varphi=\varphi_{0}(s)+\delta \varphi_{1}(s, y, \tau)+\delta^{2} \varphi_{2}(s, y, \tau)+\cdots, \\
& c_{*}=c_{0}+\delta c_{1}+\delta^{2} c_{2}+\cdots,
\end{aligned}
$$

and plug these expansions into (2.2). We obtain the following equations up to $O\left(\delta^{2}\right)$ : 
$O(1)$

$$
\varphi_{0, s s}+c_{0} \varphi_{0, s}+f\left(\varphi_{0}\right)=0
$$

$O(\delta)$

$$
\varphi_{1, s s}+\varphi_{1, y_{1} y_{1}}+c_{0} \varphi_{1, s}-\varphi_{1, \tau}+f^{\prime}\left(\varphi_{0}\right) \varphi_{1}=-\left(c_{1}+w_{1}\left(y_{1}, \tau\right)\right) \varphi_{0, s},
$$

$O\left(\delta^{2}\right)$,

$$
\begin{array}{r}
\varphi_{2, s s}+\varphi_{2, y_{1} y_{1}}+c_{0} \varphi_{2, s}-\varphi_{2, \tau}+f^{\prime}\left(\varphi_{0}\right) \varphi_{2} \\
=-c_{1} \varphi_{1, s}-c_{2} \varphi_{0, s}-w_{1}(y, \tau) \varphi_{1, s}-w_{2}(y, \tau) \varphi_{0, s}-1 / 2 f^{\prime \prime}\left(\varphi_{0}\right) \varphi_{1}^{2}
\end{array}
$$

From (2.7), we get $\varphi_{0}=\varphi_{0}(s), \varphi_{0}(0)=1 / 2$, and $c_{0}=c_{0}(f)$, the solution of the usual traveling wave equation:

$$
\begin{array}{r}
\varphi^{\prime \prime}+c \varphi^{\prime}+f(\varphi)=0, \\
\varphi(-\infty)=0, \varphi(0)=1 / 2, \varphi(+\infty)=1 .
\end{array}
$$

Solvability condition of $(2.8)$ is

$$
\int_{R^{1} \times[0,2 \pi] \times[0, T]}\left(c_{1}+w_{1}\left(y_{1}, \tau\right)\right) \varphi_{0, s} \psi d s d y_{1} d \tau=0,
$$

where $\psi=e^{c_{0} s} \varphi_{0, s}$; thus $c_{1}=-\left\langle w_{1}\left(y_{1}, \tau\right)\right\rangle=0$. We use $\langle\cdot\rangle$ to denote the average of the function inside the bracket over $[0,2 \pi] \times[0, T]$. Equation (2.8) then becomes

$$
\varphi_{1, s s}+\varphi_{1, y_{1} y_{1}}+c_{0} \varphi_{1, s}+f^{\prime}\left(\varphi_{0}\right) \varphi_{1}-\varphi_{1, \tau}=-w_{1}\left(y_{1}, \tau\right) \varphi_{0, s} .
$$

Denoting the $L^{2}$ inner product by $(\cdot, \cdot)$ and using solvability condition for $(2.9)$, we find

$$
c_{2}\left(\varphi_{0, s}, \psi\right)+\left(w_{1} \varphi_{1, s}, \psi\right)+\frac{1}{2}\left(f^{\prime \prime}\left(\varphi_{0}\right) \varphi_{1}^{2}, \psi\right)+\left(w_{2} \varphi_{0, s}, \psi\right)=0,
$$

where the last term on the left-hand side is equal to zero due to $w_{2}$ being mean zero, so

$$
c_{2}\left(\varphi_{0, s}, \psi\right)=-\frac{1}{2}\left(f^{\prime \prime}\left(\varphi_{0}\right) \varphi_{1}^{2}, \psi\right)-\left(w_{1} \varphi_{1, s}, \psi\right) .
$$

Let us solve (2.10) by Fourier series. If we define $Z_{0}^{2}=Z^{2} \backslash\{(0,0)\}$, then

$$
w_{1}\left(y_{1}, \tau\right)=\sum_{Z_{0}^{2}} b_{m, l} e^{i m y_{1}+i \omega l \tau}, \quad b_{-m,-l}=\overline{b_{m, l}},
$$

and we write

$$
\varphi_{1}\left(s, y_{1}, \tau\right)=\sum_{Z_{0}^{2}} a_{m, l}(s) e^{i m y_{1}+i \omega l \tau} .
$$

Substituting it into (2.10) gives

$$
a_{m, l}^{\prime \prime}+c_{0} a_{m, l}^{\prime}+\left(f^{\prime}\left(\varphi_{0}\right)-m^{2}-i l \omega\right) a_{m, l}=-b_{m, l} \varphi_{0, s} .
$$


Note that $\varphi_{0, s}$ satisfies

$$
a_{m, l}^{\prime \prime}+c_{0} a_{m, l}^{\prime}+f^{\prime}\left(\varphi_{0}\right) a_{m, l}=0 .
$$

So (2.13) is easily seen to admit a unique solution:

$$
a_{m, l}=\frac{b_{m, l}}{m^{2}+i l \omega} \varphi_{0, s}
$$

thus

$$
\begin{aligned}
\left\langle\varphi_{1}^{2}\right\rangle & =\sum_{Z_{0}^{2}} \frac{\left|b_{m, l}\right|^{2}}{m^{4}+l^{2} \omega^{2}} \varphi_{0, s}^{2} \\
& \equiv \beta \varphi_{0, s}^{2} .
\end{aligned}
$$

Now let $I=-\frac{1}{2} \int_{R^{1}} f^{\prime \prime}\left(\varphi_{0}\right) \varphi_{0, s}^{3} e^{c_{0} s} d s, J=-\left(w_{1} \varphi_{1, s}, \psi\right)$; then

$$
c_{2}=\frac{\beta}{\alpha} I+\frac{J}{\alpha},
$$

where $\alpha=\int_{s} \varphi_{0, s}^{2} e^{c_{0} s} d s$. By (2.14), we have

$$
\begin{array}{r}
w_{1}\left(y_{1}, \tau\right)=\sum_{Z_{0}^{2}} b_{m, l} e^{i m y_{1}+i l \omega \tau}, \\
\varphi_{1}\left(s, y_{1}, \tau\right)=\varphi_{0, s} \sum_{Z_{0}^{2}} \frac{b_{m, l}}{m^{2}+i l \omega} e^{i m y_{1}+i l \omega \tau},
\end{array}
$$

so

$$
\begin{aligned}
J & =-\left(w_{1} \varphi_{1, s}, \psi\right)=-\int_{R^{1}}\left\langle w_{1} \varphi_{1, s}\right\rangle \psi d s \\
& =-\int_{R^{1}}\left(\sum_{Z_{0}^{2}} \frac{\left|b_{m, l}\right|^{2}}{m^{2}-i l \omega}\right) \varphi_{0, s s} \varphi_{0, s} e^{c_{0} s} d s \\
& =-\int_{R^{1}}\left(\sum_{m \neq 0} \frac{\left|b_{m, 0}\right|^{2}}{m^{2}}+\sum_{m>0, l>0}\left|b_{m, l}\right|^{2} \frac{2 m^{2}}{m^{4}+l^{2} \omega^{2}}\right) \varphi_{0, s s} \varphi_{0, s} e^{c_{0} s} d s .
\end{aligned}
$$

Let

$$
\gamma=\sum_{m \neq 0} \frac{\left|b_{m, 0}\right|^{2}}{m^{2}}+\sum_{m>0, l>0}\left|b_{m, l}\right|^{2} \frac{2 m^{2}}{m^{4}+l^{2} \omega^{2}}
$$

then

$$
\begin{aligned}
J & =-\gamma \int_{R^{1}} \varphi_{0, s s} \varphi_{0, s} e^{c_{0} s} d s=-\frac{\gamma}{2} \int_{R^{1}}\left(\varphi_{0, s}^{2}\right)_{s} e^{c_{0} s} d s \\
& =\frac{\gamma}{2} c_{0} \int_{R^{1}} \varphi_{0, s}^{2} e^{c_{0} s} d s=\frac{\gamma}{2} \alpha c_{0}
\end{aligned}
$$

therefore

$$
c_{2}=\frac{\beta}{\alpha} I+\frac{\gamma}{2} c_{0}
$$


Now we show that $I=0$. In fact, differentiating (2.7) twice, we have the identity (superscripts denoting number of $s$ derivatives):

$$
L \varphi_{0, s s} \equiv \varphi_{0, s s}^{(2)}+c_{0} \varphi_{0, s s}^{(1)}+f^{\prime}\left(\varphi_{0}\right) \varphi_{0, s s}=-f^{\prime \prime}\left(\varphi_{0}\right)\left(\varphi_{0, s}\right)^{2} .
$$

Then using the property that $L^{*}\left(\varphi_{0, s} e^{c_{0} s}\right)=0, L^{*}$ being the adjoint operator of $L$, we deduce that the right-hand side of (2.17) is orthogonal to $\varphi_{0, s} e^{c_{0} s}$, which is $I=0$. It follows that $c_{2}=\frac{\gamma}{2} c_{0}$ and

$$
c_{*}=c_{0}\left(1+\frac{\gamma}{2} \delta^{2}+\text { h.o.t. }\right) .
$$

Formula (2.18) says that bistable front propagation in mean zero spatiallytemporally periodic shear is faster than that in a mean zero spatially periodic shear $\left(b_{m, l}=0, \forall l \neq 0\right)$. The enhancement is, however, monotonely decreasing with increasing temporal frequency $\omega$, showing that the direction switching in time slows down fronts. Note that the above derivation does not use the cubic form of $f$.

2.2. Other nonlinearities and front speeds. For $C^{2}$ combustion nonlinearity with cutoff, type (4), the calculations are similar, except that the linear operators are invertible in a weighted $L^{2}$ space [23]. The vanishing of integral $I$ is true also. Notice that $\gamma$ is independent of nonlinearity and depends only on the shear. Up to $O\left(\delta^{2}\right), c_{*}$ is the same for bistable and type (4) nonlinearities. In the case of types (3) and (5), one needs only to verify that the integral $\int \varphi_{0, s}^{2} e^{c_{0} s} d s$ converges to reach the same conclusion (e.g., true for $m=2$ ). We now show that formula (2.18) remains true for KPP.

To this end, we notice that the calculations for the bistable and combustion cases are no longer valid for KPP; e.g., the integral $\int \varphi_{0, s}^{2} e^{c_{0} s} d s$ diverges for KPP minimal fronts; clearly, $I$ cannot be zero either. The calculation can be done instead using variational characterization of KPP minimal speed [30]. The KPP minimal speed

$$
c_{*}=\inf _{\lambda>0} \frac{H(-\lambda)}{\lambda}
$$

where $H=H(\lambda)=\lambda^{2}+\eta(\lambda), \eta$ being the principle eigenvalue of the parabolic operator $\partial_{y y} \cdot-\partial_{\tau} \cdot+\lambda \delta w(y, \tau) \cdot$ on the torus $T_{y, \tau}^{2}$. For $\delta$ small, the principle eigenfunction $\psi$ has the expansion $\psi \sim 1+\delta \psi_{1}+\delta^{2} \psi_{2}+$ h.o.t., and $\eta=\delta^{2} \eta_{2}+$ h.o.t. The equation at $O(\delta)$ is

$$
\psi_{1, y y}-\psi_{1, \tau}=-\lambda w_{1}(y, \tau)
$$

and at $O\left(\delta^{2}\right)$ is

$$
\psi_{2, y y}-\psi_{2, \tau}+\lambda w_{1} \psi_{1}=\eta_{2}
$$

Solvability condition of (2.21) says that $\eta_{2}=\lambda\left\langle w_{1} \psi_{1}\right\rangle$. Equation (2.20) can be solved by Fourier series for $\psi_{1}$, then used to express $\eta_{2}$; the upshot is that $\eta_{2}=\lambda^{2} \gamma$, with $\gamma$ given by (2.15). Now, up to $O\left(\delta^{2}\right)$,

$$
c_{*}=\inf _{\lambda>0}\left[f^{\prime}(0) / \lambda+\left(1+\delta^{2} \gamma\right) \lambda\right]=c_{0}\left(1+\frac{\gamma}{2} \delta^{2}\right),
$$

$c_{0}=2 \sqrt{f^{\prime}(0)}$, which is in the form of $(2.18)$. 
3. Front speeds in large amplitude shears. For large amplitude shears, we show lower bounds linearly growing in shear amplitude if $f(u) \geq 0$ for $u \in[0,1]$. The upper bound of the type $c_{0}+\|W\|_{\infty}$ is straightforward by comparison principle. We generalize the method of [14] for the spatially dependent shear flows. Derivation of a lower bound is based on the properties that $\varphi_{s}>0,0<\varphi<1$, where $\varphi$ approaches zero or one with exponential rates as $s \rightarrow \pm \infty$. We state the following theorem.

Theorem 3.1 (existence, uniqueness, monotonicity). Consider continuous shear flow $\left(0, w\left(y_{1}, t\right)\right), w$ being $(2 \pi, T)$ periodic with $\int_{0}^{2 \pi} \int_{0}^{T} w\left(y_{1}, \tau\right) d y_{1} d \tau=0$, and let the nonlinearity $f$ be type (4). Then there exists a classical solution $\varphi(s, y, \tau)$ of $(2.2)$, which is unique up to a constant shift in the s variable and strictly monotone in $s$. Moreover, $0<\varphi<1$ for any $(s, y, \tau), \varphi$ tends to 0 or 1 exponentially fast in $s$, and the wave speed $c_{*} \leq-C_{w}<0$, where constant $C_{w}$ depends only on $w\left(y_{1}, \tau\right), T$, and $f$.

The proof is outlined in the appendix.

Proposition 3.1 (linear growth). Consider traveling front solutions in Theorem 3.1, and assume in addition that $\int_{0}^{2 \pi} w\left(y_{1}, \tau\right) d y_{1}=0$, for any $\tau$. If the shear field is magnified by a factor $A \gg 1$, then $c_{*}=O(A)$.

Proof. Integrate $(2.2)$ over $R^{1} \times(0,2 \pi) \times(0, T) \equiv R^{1} \times \Omega$ to get

$$
-c_{*}|\Omega|=\int_{R^{1} \times \Omega} f(\varphi) d s d y_{1} d \tau
$$

$c_{*}<0$. Multiply $(2.2)$ by $\varphi$; then integrate over $R^{1} \times \Omega$ to get $(\varphi \in(0,1), f(\varphi) \geq 0)$ :

$$
\begin{aligned}
-c_{*}|\Omega| / 2 & =-\int_{R^{1} \times \Omega}\left|\nabla_{s, y_{1}} \varphi\right|^{2} d s d y_{1} d \tau+\int_{R^{1} \times \Omega} \varphi f(\varphi) d s d y_{1} d \tau \\
& \leq-\int_{R^{1} \times \Omega}\left|\nabla_{s, y_{1}} \varphi\right|^{2} d s d y_{1} d \tau-c_{*}|\Omega|
\end{aligned}
$$

implying

$$
\int_{R^{1} \times \Omega}\left|\nabla_{s, y_{1}} \varphi\right|^{2} d s d y_{1} d \tau \leq-c_{*}|\Omega| / 2
$$

By $\varphi_{s}>0$, and (3.1)-(3.2),

$$
\begin{aligned}
& \int_{0}^{1} \sqrt{2 f\left(s^{\prime}\right)} d s^{\prime}=\frac{1}{|\Omega|} \int_{R^{1} \times \Omega} \sqrt{2 f(\varphi)} \varphi_{s} d s d y_{1} d \tau \\
\leq & \frac{1}{|\Omega|}\left(2 \int_{R^{1} \times \Omega} f(\varphi) d s d y d \tau \int_{R^{1} \times \Omega}\left|\varphi_{s}\right|^{2} d s d y_{1} d \tau\right)^{1 / 2} \leq\left|c_{*}\right| .
\end{aligned}
$$

Finally, we multiply $(2.2)$ by $\chi\left(y_{1}, \tau\right) f(\varphi)$ and integrate, where $\chi$ solves $\chi_{y_{1}, y_{1}}-\chi_{\tau}=$ $-w\left(y_{1}, \tau\right)$ subject to periodic boundary conditions. Solution $\chi$ is easily obtained by Fourier series and can be made mean zero. We find

$$
\begin{aligned}
& 0=\int_{R^{1} \times \Omega}\left(-\left|\nabla_{s, y_{1}} \varphi\right|^{2} \chi f^{\prime}(\varphi)-\chi_{y_{1}} \varphi_{y_{1}} f(\varphi)+\chi f(\varphi)^{2}\right) \\
& -\int_{R^{1} \times \Omega} \chi(F(\varphi))_{\tau}+F(1) \int_{\Omega} w \chi,
\end{aligned}
$$


where $F(u)=\int_{0}^{u} f\left(s^{\prime}\right) d s^{\prime}, u \in(0,1)$. In the last term of (3.4),

$$
F(1) \int_{\Omega} w \chi=F(1) \int_{\Omega}\left|\chi_{y_{1}}\right|^{2} .
$$

The second to last term is estimated as follows. Notice that $(F(\varphi))_{\tau}=(G(\varphi-1))_{\tau}$, where $G(u)=\int_{0}^{u} f\left(s^{\prime}+1\right) d s^{\prime}, u \in(-1,0)$. Let $\chi_{0}\left(y_{1}, \tau\right)=\int_{0}^{y_{1}} w\left(y_{1}, \tau\right) d y_{1}$, which is periodic and bounded in $L^{\infty}$ by our assumption on $w$. Then

$$
\begin{aligned}
& \int_{R^{1} \times \Omega} \chi(F(\varphi))_{\tau} \\
& =-\int_{R_{-}^{1} \times \Omega} \chi_{\tau} F(\varphi)-\int_{R_{+}^{1} \times \Omega} \chi_{\tau} G(\varphi-1) \\
& =-\int_{R_{-}^{1} \times \Omega}\left(\chi_{0}+\chi_{y_{1}}\right)_{y_{1}} F(\varphi)-\int_{R_{+}^{1} \times \Omega}\left(\chi_{0}+\chi_{y_{1}}\right)_{y_{1}} G(\varphi-1) \\
& =\int_{R_{-}^{1} \times \Omega}\left(\chi_{0}+\chi_{y_{1}}\right) \varphi_{y_{1}} f(\varphi)+\int_{R_{+}^{1} \times \Omega}\left(\chi_{0}+\chi_{y_{1}}\right) \varphi_{y_{1}} f(\varphi) \\
& =\int_{R^{1} \times \Omega}\left(\chi_{0}+\chi_{y_{1}}\right) \varphi_{y_{1}} f(\varphi) \\
& \leq\left(\left\|\chi_{0}\right\|_{\infty}+\left\|\chi_{y_{1}}\right\|_{\infty}\right)\left\|\varphi_{y_{1}}\right\|_{2}\|f(\varphi)\|_{2} .
\end{aligned}
$$

We have from (3.5) and (3.4) that

$$
\begin{aligned}
& F(1) \int_{\Omega}\left|\chi_{y_{1}}\right|^{2} d y_{1} d \tau \leq \int_{R^{1} \times \Omega}\left(\left|\nabla_{s, y_{1}} \varphi\right|^{2} \chi f^{\prime}(\varphi)+\chi_{y_{1}} \varphi_{y_{1}} f(\varphi)-\chi f(\varphi)^{2}\right) \\
& +\left(\left\|\chi_{0}\right\|_{\infty}+\left\|\chi_{y_{1}}\right\|_{\infty}\right)\left\|\varphi_{y_{1}}\right\|_{2}\|f(\varphi)\|_{2} \\
& \leq \frac{\left|c_{*}\right||\Omega|}{2}\|\chi\|_{\infty}\left\|f^{\prime}\right\|_{\infty}+\frac{\left|c_{*}\right||\Omega|}{2^{1 / 2}}\left\|\chi_{y_{1}}\right\|_{\infty}\|f\|_{\infty}^{1 / 2}+\left|c_{*}\|\Omega \mid\| \chi\left\|_{\infty}\right\| f \|_{\infty}\right. \\
& +|\Omega|\left(\left\|\chi_{0}\right\|_{\infty}+\left\|\chi_{y_{1}}\right\|_{\infty}\right)\left|c_{*}\right|\|f\|_{\infty}^{1 / 2},
\end{aligned}
$$

implying the lower bound

$$
\begin{gathered}
\left|c_{*}\right| \geq \frac{\left\|\chi_{y_{1}}\right\|_{2}^{2} F(1)}{|\Omega|} \underline{B}^{-1} \\
\underline{B}=\frac{1}{2}\|\chi\|_{\infty}\left|f^{\prime}\right|_{\infty}+\left(1+2^{-1 / 2}\right)\left\|\chi_{y_{1}}\right\|_{\infty}\|f\|_{\infty}^{1 / 2}+\|\chi\|_{\infty}\|f\|_{\infty}+\left\|\chi_{0}\right\|_{\infty}\|f\|_{\infty}^{1 / 2} .
\end{gathered}
$$

We see that for large amplitude shear flows, i.e., replacing $w$ by $A w\left(y_{1}, \tau\right), A$ being a large parameter, the front speed $c_{*} \geq O(A)$. Combining with the upper bound $\left|c_{*}\right| \leq\left|c_{0}\right|+A\|w\|_{\infty}$, we have $c_{*}=O(A), A \gg 1$.

We remark that if the minimal speed traveling fronts exist for types (2), (3), and (5) nonlinearities and share the qualitative properties as for type (4), the above proof works also to yield $O(A)$ speed growth. The existence of such fronts for types (1), (2), (3), and (5) is left for future publications. Instead, we shall demonstrate numerically in the next section that both the quadratic speed enhancement for small shears and linear speed growth for large shears are observed for the types (1), (2), and (3) with $m=2$, and for (4). Comparison principle implies that the results also hold for type (3) at any $m>2$, and for type (5), in the large $A$ regime. 


\section{Numerical results on front speeds.}

4.1. Numerical method. We use an explicit second order finite difference method to compute the front solutions to the equation

$$
u_{t}=\alpha \Delta_{x, y} u+\delta b(y, t) u_{x}+f(u)
$$

where $\alpha, \delta>0, b(y, t)$ being a spatially-temporally periodic function with mean zero in $y$ for any $t ;(x, y) \in \Omega=\left(0, X_{f}\right) \times\left(0, Y_{f}\right)$, with zero Neumann boundary condition and front like initial data (e.g., characteristic function over $\left[0, X_{0}\right) \times\left(0, Y_{f}\right), X_{0}<$ $\left.X_{f}\right)$. The $\Delta_{x, y} u$ term is discretized with standard second order central differencing. The advection term $\delta b(y, t) u_{x}$ is discretized with second order upwind and Van Leer limiter [8], which is chosen because $\alpha$ typically is small in our simulations; e.g., $\alpha=$ 0.025. Time stepping is the two-step second order Adams-Bashforth scheme [12]. The Neumann boundary condition is discretized by one-sided second order differencing. The time step is small enough to maintain numerical stability and accuracy for chosen spatial grids. Once solutions are computed, we find the average front location in $x$ by integrating the solution over the entire domain and dividing by the domain width:

$$
I(t)=\frac{1}{Y_{f}} \int_{\Omega} u(x, y, t) d x d y .
$$

We then approximate the average front speed $c^{*}(\delta)$ by

$$
c_{*}(\delta) \sim \frac{I\left(t_{f}\right)-I\left(t_{1}\right)}{t_{f}-t_{1}}, \quad t_{1}<t_{f} .
$$

We take $t_{1}$ suitably large to allow time for the front to form and choose $t_{f}$ large enough for the resulting speeds to stabilize. The integral $I$ in (4.2) is computed with the composite Simpson's rule.

4.2. Numerical parameters. The function $b(y, t)$ is of the form

$$
b(y, t)=(1+\sin (\omega t)) \sin (10 y),
$$

and we vary the shear amplitude $\delta$ and frequency $\omega$. For small amplitude shears, the computational domain consisted of $875 \times 157$ grid points with grid size $d x=d y=0.04$, corresponding to the domain of $(0,35) \times(0,2 \pi)$. The diffusion constant was chosen to be $\alpha=0.025$, which produced moderately steep front profiles.

For larger amplitudes $\delta$, however, the faster front speeds necessitated a larger computational domain. The grid points reach $2000 \times 157$ with grid size $d x=d y=0.05$, corresponding to the numerical domain of $(0,100) \times(0,2 \pi)$. For each simulation, the discrete time step is $d t=0.004$. The initial data is $u_{0}(x)=1, x \leq 10$, $u_{0}(x)=0, x>10$. Each of the samples was evolved in time until $t_{f}=30$, at which point the front speed is stabilized and estimated using (4.3) with $t_{1}=5$. With these parameters, the effect of numerical diffusion does not significantly change the results, thanks to the second order accuracy of the method in both time and space. Further grid refinement (e.g., $d x \rightarrow d x / 2, d t \rightarrow d t / 4$ ) shows that changes of front speeds are under $0.4 \%$.

The following nonlinear functions are used in the simulation:

(1) bistable nonlinearity: $f(u)=u(1-u)(u-0.25)$;

(2) combustion nonlinearity: $f(u)=(1-u)(u-0.5)$ if $u>0.5 ; f(u)=0$ if $u \leq 0.5$;

(3) KPP nonlinearity: $f(u)=u(1-u)$;

(4) Zeldovich nonlinearity: $f(u)=u^{2}(1-u)$. 
4.3. Numerical results. We are interested in testing how $c_{*}(\delta)-c_{0}$ scales with $\delta^{p}$, especially whether $p$ is near 2 for $\delta$ small and $p=1$ for $\delta$ large. Using the numerically calculated speeds $c_{0}$ and $c_{*}(\delta)$, we determined the exponents $p$ using the least squares method to fit a line to a log-log plot of speed versus amplitude. That is, we determined the slope of the best-fit line through the data points $\left(\log (\delta), \log \left(c(\delta)-c_{0}\right)\right)$ for each shear amplitude $\delta$. For each nonlinearity we computed the exponent $p$ for both the small amplitude and the large amplitude regimes, using a variety of frequencies for the time dependent shear. For the small amplitude regime, $\delta$ values range from 0.005 to 0.10 with increment 0.005 . For the large amplitude regime, $\delta$ values range from 3.5 to 4.0 with increment 0.10 . The exponent is observed to converge at and beyond this range of $\delta$.

The calculated exponents are shown in Tables 1-4 below for each nonlinearity and a sequence of temporal frequencies. These results show that with each nonlinearity, $c_{*}(\delta)-c_{0} \sim O\left(\delta^{2}\right)$ when the amplitude is small, and $c_{*}(\delta) \sim O(\delta)$ when the amplitude is large. These results agree with and complement our analytical findings. In particular, the growth of large amplitude bistable front speeds in shears shows an interesting contrast with the bistable front quenching (propagation failure) phenomena when either the reaction or the diffusion coefficients become strongly heterogeneous [29].

TABLE 1

Case 1: Bistable nonlinearity.

\begin{tabular}{|c|c|c|}
\hline & $\delta \ll 1$ & $\delta \gg 1$ \\
\hline Frequency $(\omega)$ & Exponent $(p)$ & Exponent $(p)$ \\
\hline 5.0 & 1.998 & 1.064 \\
\hline 10.0 & 1.999 & 1.069 \\
\hline 15.0 & 1.999 & 1.070 \\
\hline 20.0 & 1.998 & 1.071 \\
\hline
\end{tabular}

TABLE 2

Case 2: Combustion nonlinearity.

\begin{tabular}{|c|c|c|}
\hline & $\delta \ll 1$ & $\delta \gg 1$ \\
\hline Frequency $(\omega)$ & Exponent $(p)$ & Exponent $(p)$ \\
\hline 5.0 & 1.995 & 1.033 \\
\hline 10.0 & 1.990 & 1.065 \\
\hline 15.0 & 1.993 & 1.067 \\
\hline 20.0 & 1.992 & 1.068 \\
\hline
\end{tabular}

TABLE 3

Case 3: KPP nonlinearity.

\begin{tabular}{|c|c|c|}
\hline & $\delta \ll 1$ & $\delta \gg 1$ \\
\hline Frequency $(\omega)$ & Exponent $(p)$ & Exponent $(p)$ \\
\hline 5.0 & 1.977 & 1.075 \\
\hline 10.0 & 1.981 & 1.080 \\
\hline 15.0 & 1.980 & 1.080 \\
\hline 20.0 & 1.996 & 1.080 \\
\hline
\end{tabular}

We also observe that for a fixed amplitude, the speed enhancement decreases with increasing temporal frequency $\omega$ of the shear. This can be observed clearly in the plots of the front speeds for larger amplitude shears.

Figure 1 shows a comparison of speeds for bistable fronts at moderately large amplitude as temporal frequency $\omega$ varies at 5, 10, 15, 20. Clearly, the speeds go 
TABLE 4

Case 4: Zeldovich nonlinearity.

\begin{tabular}{|c|c|c|}
\hline & $\delta \ll 1$ & $\delta \gg 1$ \\
\hline Frequency $(\omega)$ & Exponent $(p)$ & Exponent $(p)$ \\
\hline 5.0 & 1.991 & 1.038 \\
\hline 10.0 & 1.991 & 1.045 \\
\hline 15.0 & 1.993 & 1.046 \\
\hline 20.0 & 1.992 & 1.047 \\
\hline
\end{tabular}

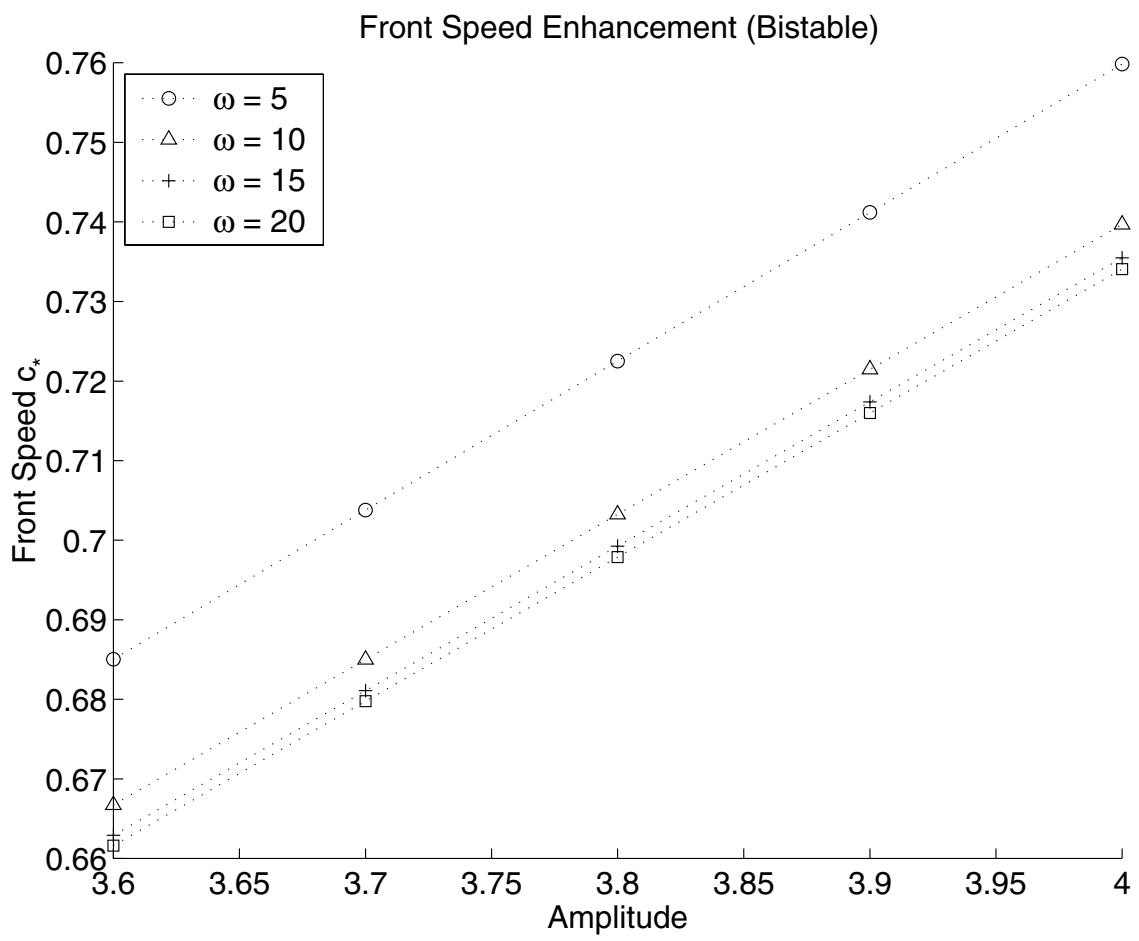

FIG. 1. Comparison of bistable front speeds at moderately large amplitudes as temporal shear frequency varies; $\omega=5,10,15,20$.

down with increasing $\omega$ and converge to a limiting value for large $\omega$. Also the slopes are the same for different $\omega$ 's, suggesting that

$$
c_{*}(\delta) \sim k_{0} \delta+k_{1}(\omega), \quad \delta \gg 1,
$$

where $k_{0}\left(k_{1}\right)$ is constant independent of (decreasing in) $\omega$.

Figure 2 shows a similar comparison of combustion front speeds at the same temporal frequency values. Figures 3 and 4 illustrate the same speed slow down with increasing $\omega$ at the small shear amplitude regime. We plot only $\omega=5,15$, as the speed curves get much closer to each other in this regime.

A plot of speed versus amplitude is in Figure 5 for bistable nonlinearity at $\omega=10$. Such curves for other nonlinearities are quite similar. Figure 6 is a representative plot for combustion nonlinearity at $\omega=15$. The transition between the two scaling regimes $(p=2$ and $p=1)$ appears in $\delta \in[0.5,1]$. 


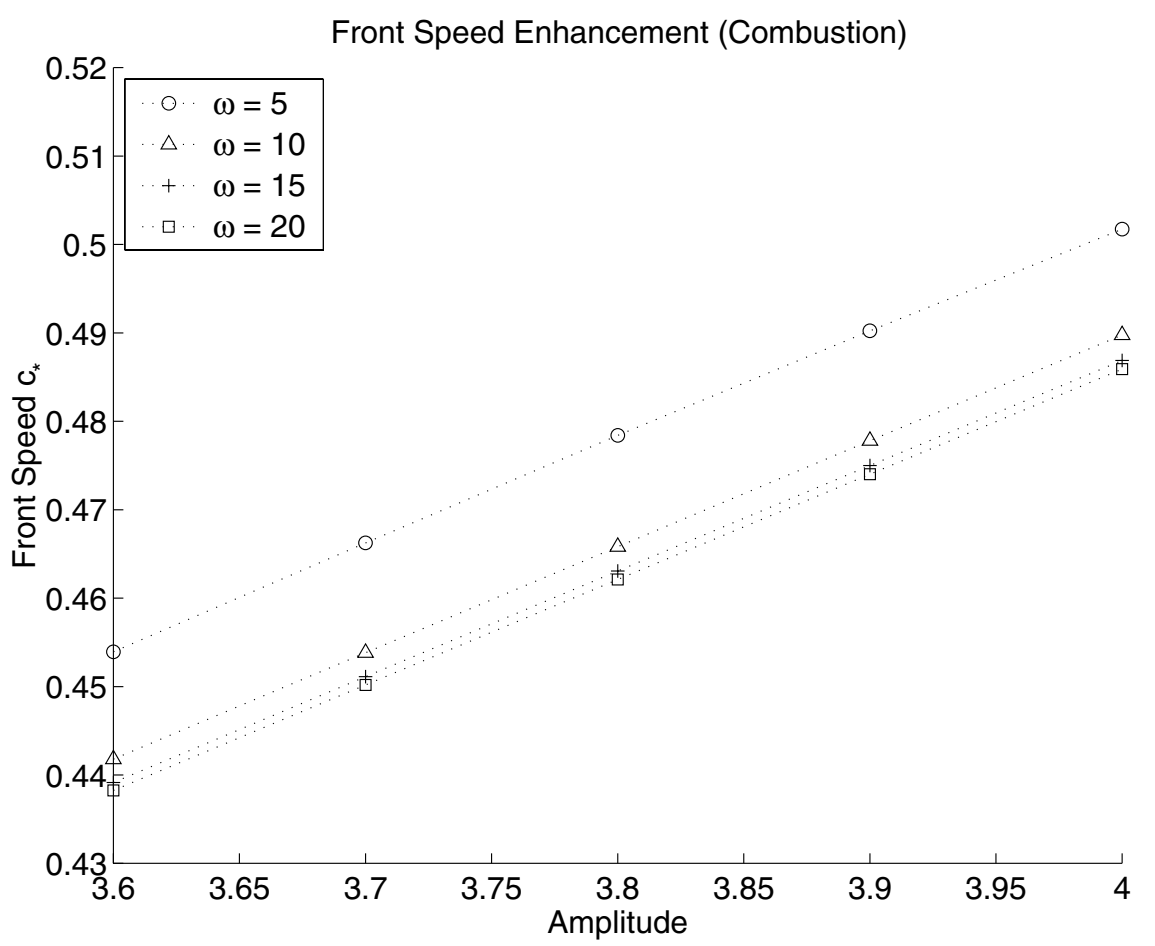

FIG. 2. Comparison of combustion front speeds at moderately large amplitudes as temporal shear frequency varies; $\omega=5,10,15,20$.

5. Conclusions. Using combined analytical and numerical methods, we find that reaction-diffusion front speeds through mean zero spatially-temporally periodic shears obey robust asymptotics in both the small and large amplitude regimes. In the small amplitude regime, the enhanced speeds are proportional to shear amplitude squared, universal to all known nonlinearities. In the large amplitude regime, the enhanced speeds scale linearly with shear amplitude, again for all known nonlinearities. In both regimes, the enhancement decreases with increasing shear temporal frequency, indicating a slowdown of front speeds under shear direction changing in time.

In future work, it will be interesting to consider spatially-temporally quasiperiodic, as well as random, shear fields and investigate efficient numerical methods that can compute large time front speeds accurately without fully resolving solutions; see [10], [6], among others.

Appendix. Traveling front existence and qualitative properties. We outline the proof of Theorem 3.1 in the case of type (4) nonlinearity. A key property is that the operator in the linear part of $(2.2)$,

$$
L \varphi \equiv \varphi_{s s}+\varphi_{y_{1} y_{1}}+\left(c_{*}+w\left(y_{1}, \tau\right)\right) \varphi_{s}-\varphi_{\tau},
$$

satisfies the strong maximum principle for $\left(s, y_{1}, \tau\right) \in R^{1} \times T^{2}$ (assume without loss of generality that $\omega=1$ ), even though locally it is parabolic. That is to say, if $L \varphi \geq(\leq) 0$ on $R^{1} \times T^{2}$, where $\varphi$ attains its maximum (minimum) at a finite point $\left(s_{0}, y_{1,0}, \tau_{0}\right)$, then $\varphi$ is identically a constant. In fact, the standard parabolic maximum principle implies that $\varphi$ is a constant for $\tau \geq \tau_{0}$, but now periodicity in $\tau$ extends 


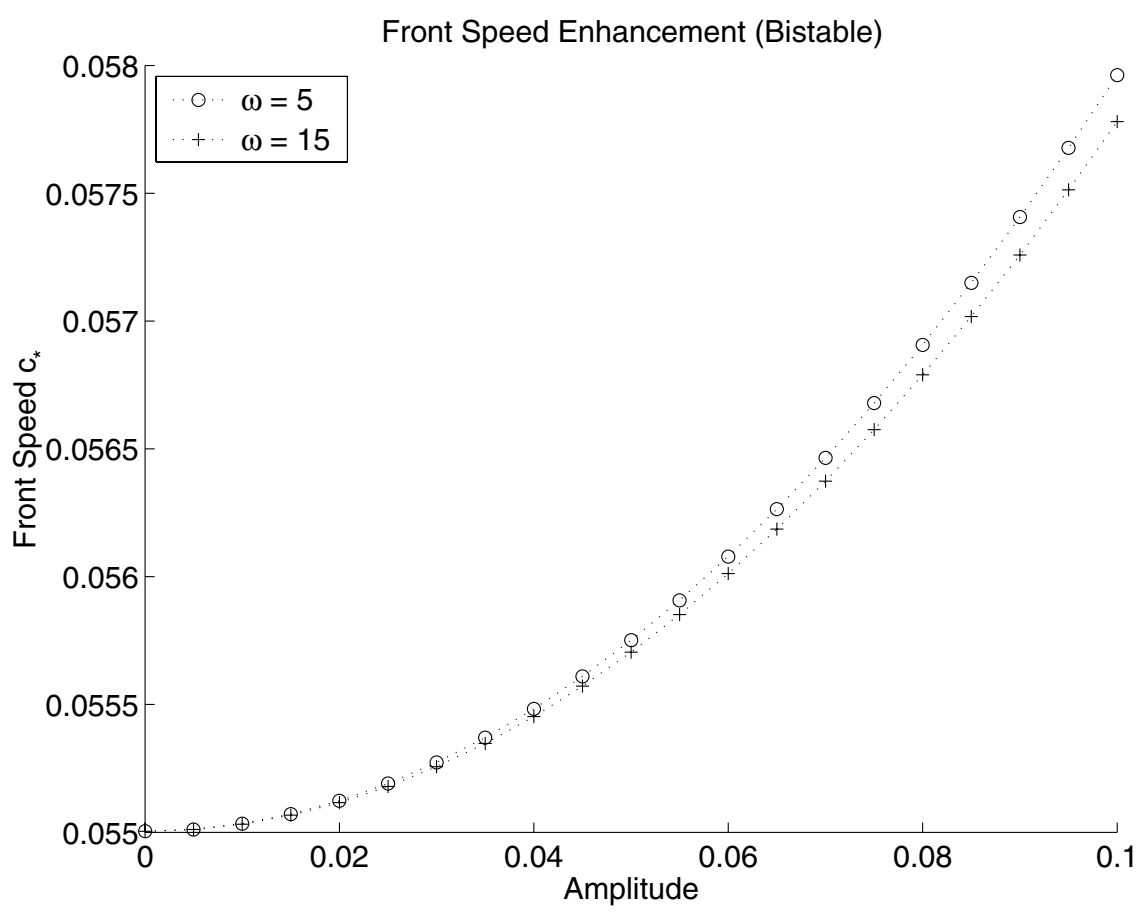

FIG. 3. Comparison of bistable front speeds at small amplitudes as temporal shear frequency varies; $\omega=5,15$.

it to all $\tau$. Based on the strong maximum principle, we apply the sliding domain method [4] to show that $\varphi_{s}>0$, and $0<\varphi<1$, and to show the uniqueness of solutions up to a constant translation in $s$ as in [27]. The existence can be established by the method of continuation as in [30]. Let us first assume that $f(u) \in C^{2}$. For small $\delta$, existence under the normalization condition $\max _{T^{2}} \psi(0, y, \tau)=\theta$ follows from contraction mapping principle [26]. Monotonicity $\varphi_{s}$ implies that the linearized operator around any solution is Fredholm and has one-dimensional kernel; hence it allows the local continuation of solution (both $\varphi$ and wave speed $c_{*}$ ) in $\delta$. To ensure the continuation to any given value of $\delta$, we show the closeness of continuation; namely, the limit of any sequence of solutions remains a solution. Compactness on any finite domain $[-M, M] \times T^{2}$ is provided by parabolicity of $L$. Additionally, we need to control $s$ at infinity. We construct upper solutions for $s \leq 0$ of the form $e^{\mu s} \psi\left(y_{1}, \tau\right)$, where $\mu>0, \psi\left(y_{1}, \tau\right)>0$ uniformly in the limit so that the limiting solution decays to zero exponentially fast as $s \rightarrow-\infty$. Such upper solutions exist as long as the wave speed $c_{*}<0$ is uniformly bounded away from zero, which we show below. Thereafter, $f(u) \geq 0$ and strong maximum principle of $L$ implies that $\psi(+\infty)=\theta$ or 1 . That $\psi=\theta$ is impossible, as otherwise $\psi$ will be constant by strong maximum principle of $L$ (contradicting its decay to zero at $-\infty$ ). Existence of global solutions follows. An approximation argument takes care of less smooth reaction nonlinearities.

Now we show that $c_{*}$ is bounded away from zero. If $w\left(y_{1}, \tau\right)$ has mean zero in $y_{1}$ for any $\tau$, this is done already in Proposition 3.1. Now with only space and time mean being zero, we shall pick up additional terms to estimate. The integral

$$
\int_{0}^{y_{1}} w(y, \tau) d y=\chi_{0}\left(y_{1}, \tau\right)+a v^{\prime}(\tau) y_{1}
$$




\section{Front Speed Enhancement (Combustion)}

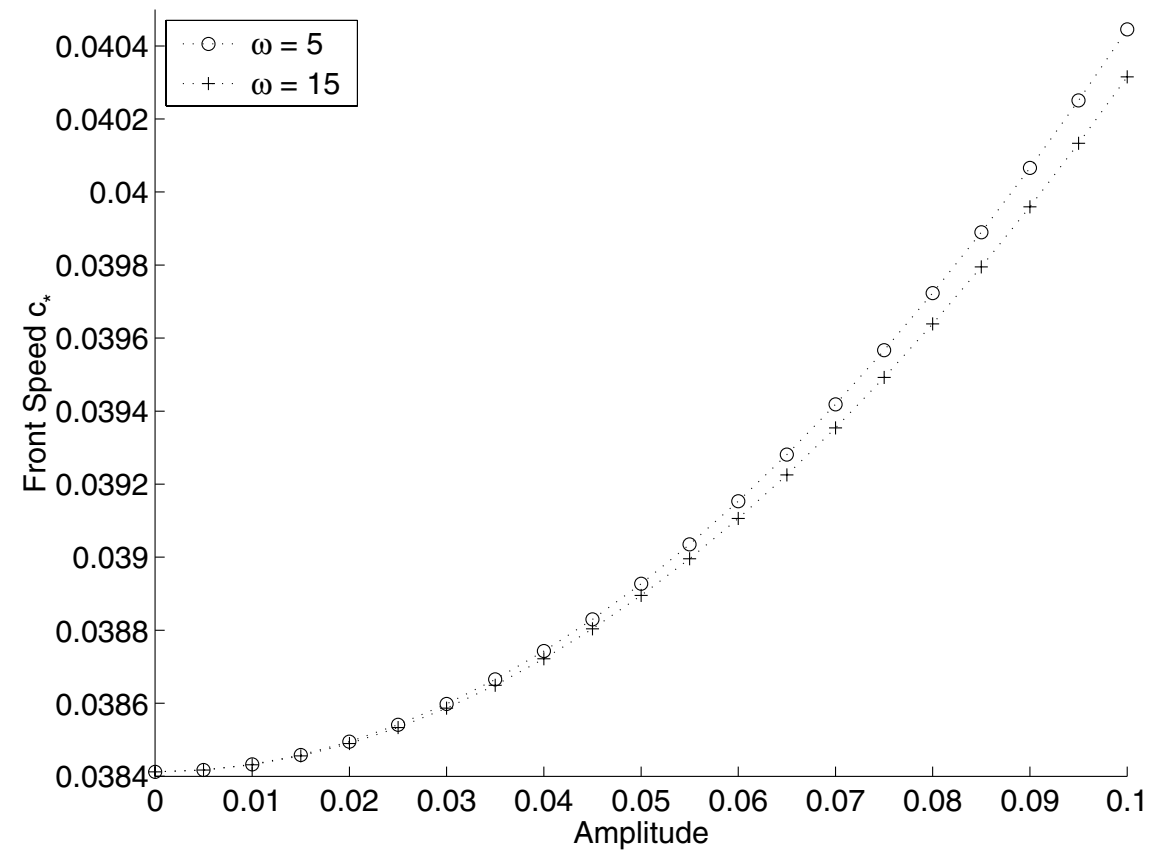

FIG. 4. Comparison of combustion front speeds at small amplitudes as temporal shear frequency varies; $\omega=5,15$.

where $\chi_{0}\left(y_{1}, \tau\right)$ and $a v=a v(\tau)$ are periodic. The estimate (3.5) becomes

$$
\begin{aligned}
& \int_{R^{1} \times \Omega} \chi(F(\varphi))_{\tau} \\
& =-\int_{R_{-}^{1} \times \Omega} \chi_{\tau} F(\varphi)-\int_{R_{+}^{1} \times \Omega} \chi_{\tau} G(\varphi-1) \\
& =-\int_{R_{-}^{1} \times \Omega}\left(\chi_{0}+a v^{\prime} y_{1}+\chi_{y_{1}}\right)_{y_{1}} F(\varphi)-\int_{R_{+}^{1} \times \Omega}\left(\chi_{0}+a v^{\prime} y_{1}+\chi_{y_{1}}\right)_{y_{1}} G(\varphi-1) \\
& =\int_{R^{1} \times \Omega}\left(\chi_{0}+\chi_{y_{1}}\right) \varphi_{y_{1}} f(\varphi)+\int_{R^{1} \times \Omega} a v(\tau) f(\varphi) \varphi_{\tau} \\
(\mathrm{A} .2) & \leq\left(\left\|\chi_{0}\right\|_{\infty}+\left\|\chi_{y_{1}}\right\|_{\infty}\right)\left\|\varphi_{y_{1}}\right\|_{2}\|f(\varphi)\|_{2}+\|a v\|_{\infty}\|f(\varphi)\|_{2}\left\|\varphi_{\tau}\right\|_{2} .
\end{aligned}
$$

Next we bound $\left\|\varphi_{\tau}\right\|_{2}$. Multiply (2.2) by $\varphi_{\tau}$, integrate over $R^{1} \times \Omega$, and integrate by parts to get

$$
\int_{R^{1} \times \Omega}\left(c_{*}+w\left(y_{1}, \tau\right)\right) \varphi_{s} \varphi_{\tau}-\int_{R^{1} \times \Omega} \varphi_{\tau}^{2}=0
$$

implying via Cauchy-Schwarz inequality that

$$
\left\|\varphi_{\tau}\right\|_{2}^{2} \leq\left(\left|c_{0}\right|+2\|w\|_{\infty}\right)\left\|\varphi_{s}\right\|_{2}\left\|\varphi_{\tau}\right\|_{2}
$$

or, in view of $(3.2)$,

(A.3) $\quad\left\|\varphi_{\tau}\right\|_{2} \leq\left(\left|c_{0}\right|+2\|w\|_{\infty}\right)\left\|\varphi_{s}\right\|_{2} \leq\left(\left|c_{0}\right|+2\|w\|_{\infty}\right)|\Omega|^{1 / 2}\left|c^{*}\right|^{1 / 2}$. 


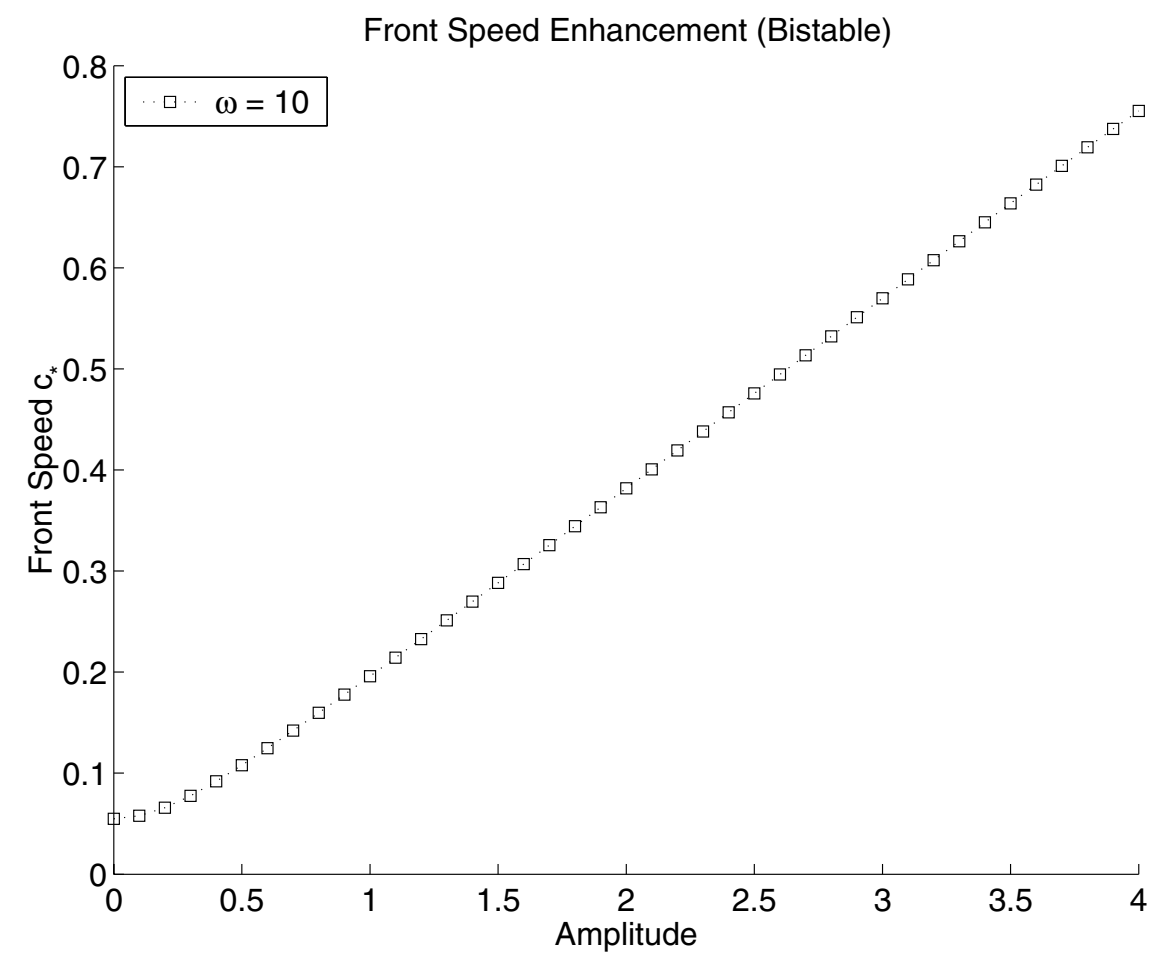

FIG. 5. Bistable front speeds versus amplitude at temporal shear frequency $\omega=10$.

Plugging (A.3) into (A.2), along with $\|f(\varphi)\|_{2} \leq\|f\|_{\infty}^{1 / 2}\left|c_{*}\right|^{1 / 2}|\Omega|^{1 / 2}$, we have the lower bound on $c_{*}$ :

$$
\begin{gathered}
\left|c_{*}\right| \geq \frac{\left\|\chi_{y_{1}}\right\|_{2}^{2} F(1)}{|\Omega|} \underline{B}^{-1} \\
\underline{B}=\frac{1}{2}\|\chi\|_{\infty}\left|f^{\prime}\right|_{\infty}+\left(1+2^{-1 / 2}\right)\left\|\chi_{y_{1}}\right\|_{\infty}\|f\|_{\infty}^{1 / 2}+\|\chi\|_{\infty}\|f\|_{\infty}+\left\|\chi_{0}\right\|_{\infty}\|f\|_{\infty}^{1 / 2} \\
(\mathrm{~A} .4)+\|f\|_{\infty}^{1 / 2}\left(\left|c_{0}\right|+2\|w\|_{\infty}\right)\|a v\|_{\infty} .
\end{gathered}
$$

We note that the monotonicity of traveling fronts implies the attractivity of front speeds among the time dependent solutions with front initial data as in [28]. Finally, we remark that uniqueness and monotonicity hold for type (1) nonlinearity, and type (1) existence for small $\delta$ follows from [26]. With additional work, as in [5], existence for any $\delta$ and for other nonlinearities can be established. However, we shall not pursue it here.

Acknowledgments. The work was partially performed during J. X.'s visit to Institut H. Poincaré (IHP) in October, 2002. J. X. would like to thank H. Berestycki and J.-M. Roquejoffre for their invitation and their hospitalities, as well as IHP for a visiting professorship.

J. X. would like to thank A. Majda and A. Bourlioux for helpful conversations on time dependent advection and their work [18], as well as S. Heinze for discussing his work [14]. 


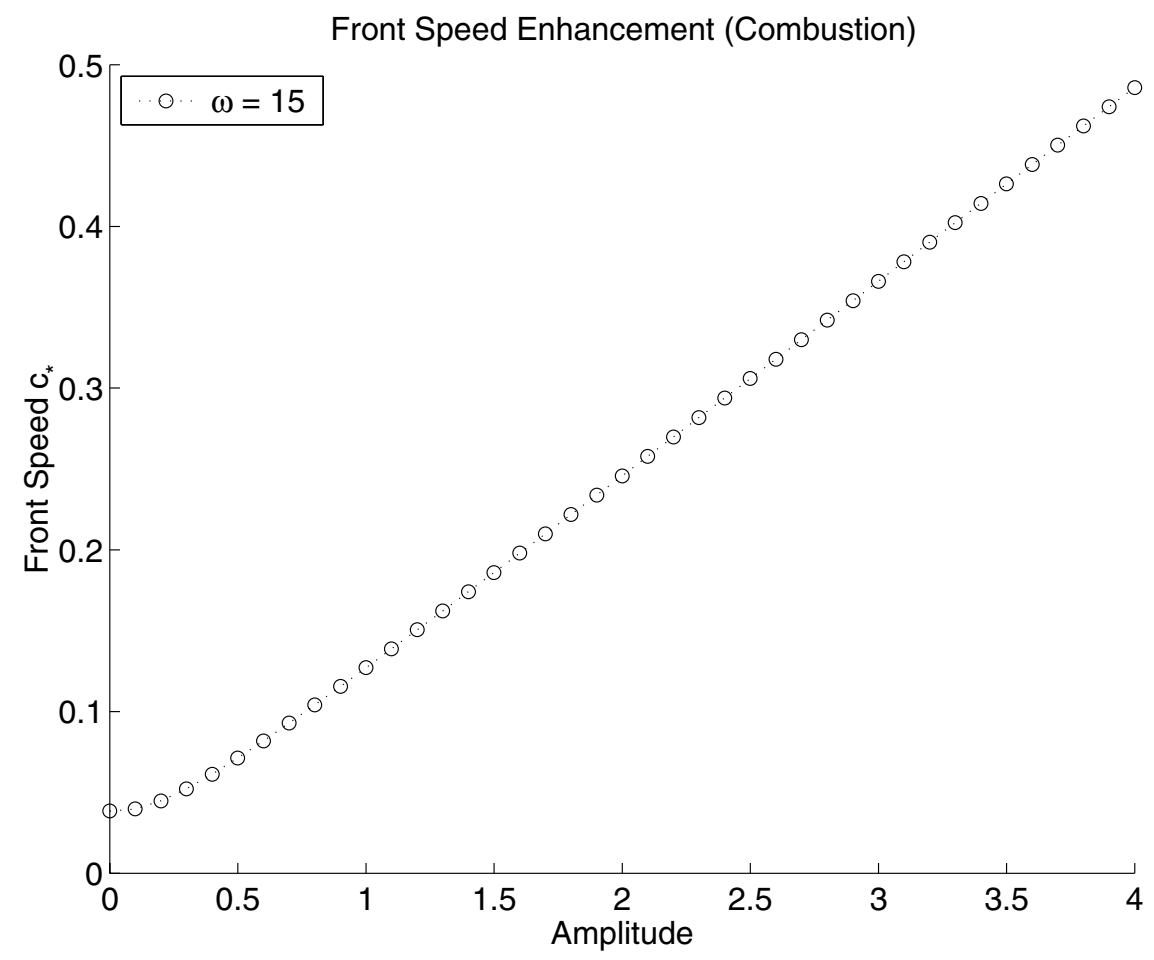

FIG. 6. Combustion front speeds versus amplitude at temporal shear frequency $\omega=15$.

\section{REFERENCES}

[1] B. Audoly, H. Berestycki, and Y. Pomeau, Réaction-diffusion en ećoulement stationnaire rapide, Note C. R. Acad. Sci. Paris Sér. II, 328 (2000), pp. 255-262.

[2] H. BERESTYCKI, The influence of advection on the propagation of fronts in reaction-diffusion equations, in Proceedings of the NATO ASI Conference, Cargese, France, H. Berestycki and Y. Pomeau, eds., Kluwer, Dordrecht, The Netherlands, to appear.

[3] H. Berestycki, B. Larrouturou, and P. L. Lions, Multi-dimensional travelling-wave solutions of a flame propagation model, Arch. Rational Mech. Anal., 111 (1990), pp. 33-49.

[4] H. Berestycki And L. Nirenberg, On the method of moving planes and the sliding method, Bol. Soc. Brasil. Mat. (N.S.), 22 (1991), pp. 1-37.

[5] H. Berestycki and L. Nirenberg, Traveling fronts in cylinders, Ann. Inst. H. Poincaré Anal. Non Linéaire, 9 (1992), pp. 497-572.

[6] L.-T. Cheng And W. E, The heterogeneous multi-scale methods for interface dynamics, in Contemporary Mathematics: Special Volume in Honour of S. Osher; S. Y. Cheng, C. W. Shu, and T. Tang, eds.; AMS, Providence, RI, to appear.

[7] P. Clavin and F. A. Williams, Theory of premixed-flame propagation in large-scale turbulence, J. Fluid Mech., 90 (1979), pp. 598-604.

[8] P. Colella, Multidimensional upwind methods for hyperbolic conservation laws, J. Comput. Phys., 87 (1990), pp. 171-200.

[9] P. Constantin, A. Kiselev, A. Oberman, and L. Ryzhik, Bulk burning rate in passivereactive diffusion, Arch. Ration. Mech. Anal., 154 (2000), pp. 53-91.

[10] W. E. B. EnGquist, The heterogeneous multi-scale methods, Commun. Math. Sci., 1 (2003), pp. $87-132$.

[11] M. Freiduin, Functional Integration and Partial Differential Equations, Ann. of Math. Stud. 109, Princeton University Press, Princeton, NJ, 1985.

[12] G. Golub and J. Ortega, Scientific Computing and Differential Equations, Academic Press, Boston, MA, 1992.

[13] B. Haslam And P. Ronney, Fractal properties of propagating fronts in a strongly stirred fluid, 
Phys. Fluids, 7 (1995), pp. 1931-1937.

[14] S. HeInze, The Speed of Travelling Waves for Convective Reaction-Diffusion Equations, Preprint 84, Max-Planck-Institut für Mathematik in den Naturewissenschaften, Leipzig, Germany, 2001.

[15] S. Heinze, G. Papanicolaou, and A. Stevens, Variational principles for propagation speeds in inhomogeneous media, SIAM J. Appl. Math., 62 (2001), pp. 129-148.

[16] A. R. Kerstein And W. T. Ashurst, Propagation rate of growing interfaces in stirred fluids, Phys. Rev. Lett., 68 (1992), p. 934.

[17] A. Kiselev AND L. RYZHIK, Enhancement of the traveling front speeds in reaction-diffusion equations with advection, Ann. Inst. H. Poincaré Anal. Non Linéaire, 18 (2001), pp. 309358.

[18] B. Khouider, A. Bourlioux, And A. Majda, Parameterizing turbulent flame speed-Part I: Unsteady shears, flame residence time and bending, Combust. Theory Model., 5 (2001), pp. 295-318.

[19] A. J. Majda And P. E. Souganidis, Large scale front dynamics for turbulent reaction-diffusion equations with separated velocity scales, Nonlinearity, 7 (1994), pp. 1-30.

[20] A. Majda And P. Souganidis, Flame fronts in a turbulent combustion model with fractal velocity fields, Comm. Pure Appl. Math., 51 (1998), pp. 1337-1348.

[21] G. Papanicolaou And J. XIn, Reaction-diffusion fronts in periodically layered media, J. Statist. Phys., 63 (1991), pp. 915-931.

[22] P. Ronney, Some open issues in premixed turbulent combustion, in Modeling in Combustion Science, Lecture Notes in Phys. 449, J. D. Buckmaster and T. Takeno, eds., SpringerVerlag, Berlin, 1995, pp. 3-22.

[23] J.-M. Roquejoffre, Stability of traveling fronts in a model for flame propagation, Part II: Nonlinear stability, Arch. Rational Mech. Anal., 117 (1992), pp. 119-153.

[24] N. Shigesada And K. Kawasaki, Biological Invasions: Theory and Practice, Oxford University Press, Oxford, UK, 1997.

[25] N. Vladimirova, P. Constantin, A. Kiselev, O. Ruchaiskiy, and L. Ryzhik, Flame Enhancement and Quenching in Fluid Flows, preprint, 2002.

[26] J. XIN, Existence and stability of travelling waves in periodic media governed by a bistable nonlinearity, J. Dynam. Differential Equations, 3 (1991), pp. 541-573.

[27] J. XIN, Existence of planar flame fronts in convective-diffusive periodic media, Arch. Rational Mech. Anal., 121 (1992), pp. 205-233.

[28] J. XIN, Existence and nonexistence of traveling waves and reaction-diffusion front propagation in periodic media, J. Statist. Phys., 73 (1993), pp. 893-926.

[29] J. Xin AND J. ZhU, Quenching and propagation of bistable reaction-diffusion fronts in multidimensional periodic media, Phys. D, 81 (1995), pp. 94-110.

[30] J. XIN, Front propagation in heterogeneous media, SIAM Rev., 42 (2000), pp. 161-230.

[31] J. XIN, KPP front speeds in random shears and the parabolic Anderson problem, Methods Appl. Anal., to appear.

[32] V. Yакнот, Propagation velocity of premixed turbulent flames, Comb. Sci. Tech., 60 (1988), p. 191.

[33] S. VAN DER ZEE AND W. VAN RIEMSDIJK, Transport of reactive solute in spatially variable soil systems, Water Resources Research, 23 (1987), pp. 2059-2069. 